\title{
OWLAN Up-Down Link Simultaneously by Optical Spin Generated
}

\author{
M. Bunruangses ${ }^{1}$, B. Piyatamrong ${ }^{2}$, S. Mitatha ${ }^{2}$ and P.P. Yupapin ${ }^{3}$ \\ ${ }^{1}$ Department of Computer Engineering, faculty of Industrial Education, \\ Rajamangala University of Technology Phra Nakhon, Bangkok, 10300, Thailand. \\ ${ }^{2}$ Hibrid Computing Research Laboratory, Faculty of Engineering, ${ }^{3}$ Nanoscale Science and Engineering Research Alliance, Faculty \\ of Science, King Mongkut's Institute of Technology Ladkrabang, Bangkok 10520, Thailand. \\ Montreeb@yahoo.com, bunjong@eau.ac.th, \{kmsomsak \& kypreech\}@kmitl.ac.th
}

\begin{abstract}
This paper proposed a new idea of optical wireless communication simultaneously using the optical spin phenomena. The spin generation from bright and dark soliton conversion behaviors within a modified optical add-drop filter known as PANDA ring resonator is proposed. The orthogonal solitons can be formed within the system and detected simultaneously at the output ports. Under the resonant condition, the dark and bright soliton pair corresponding to the left-hand and right-hand rotating solitons (photons) can be generated. When a soliton is absorbed by an object, an angular momentum of either $+\hbar$ or $-\hbar$ is imparted to the object, in which two possible spin states known as optoelectronic(soliton) spins are exhibited. The arrays of orthogonal soliton spins can be applied a spin up for uplink and spin down for downlink representation in optical wireless communication.
\end{abstract}

Index Terms-soliton,uplink,downlink,spintronics,optical wireless

\section{Introduction.}

Indoor optical wireless [1] have been applying many applications, ranging from simple remote controls in home to more complex wireless local area networks. Many other applications are envisaged for the future, including data networking in the indoor environment and the delivery of broadband multimedia services to mobile users within such an environment together with general connectivity. The optical wireless communication refers to use infrared radiation (IR) radiate wireless signal among nodes which can be providing communication high data bit rates. The indoor optical wireless was technologies of IR by used laser diodes (LDs), light emitting diodes (LEDs), and photo-diodes are transmitter and receiver respectively. The IR signal does not penetrate walls, thus providing a degree of privacy within the office area. In addition to privacy, this feature of IR systems makes it easier to build a cell-based network. For example, in an office building each room would be a cell and there would be no interference between the cells. Therefore, all units can be identical in a cellular architecture as compared to RF configuration in which the operating frequencies of neighboring cells have to be different. Due to above reasons, optical wireless systems are becoming more popular in various operating environments, such as houses, offices, medical facilities, manufacturing plants, and business establishments.

Optical wireless LANs (OWLAN) is the ones application for optical wireless which uses IR technology and supports the required mobility within buildings which operate in the operating in the infrared $(850 \mathrm{~nm})$ normally. OWLAN propagation optical signals inside a room by used three basic propagation modes are diffuse infrared, directed beam infrared (P2P), and quasi-diffused infrared. The OWLAN exchanged data by modulation technique which transmitted information is coded as waveforms and then these waveforms modulate signal intensity (amplitude) of emitted infrared light such as On-Off-Keying (OOK), Pulse Modulation (PM) and Subcarrier Modulation [2, 3]. These techniques bring optical wireless signal to optical wireless receivers and detect with optical filter by using thin dielectric film such as Fabry-Perot resonator or silicon detector (GaAs). In the recently, optical add-drop filters have been widely investigated in many area of applications, especially, when the use of an all-optical device which is formed by the modified add-drop optical filter.

The basic add-drop filter proposed by Mitatha et al $[4,5]$, applied for simultaneous wireless up-down link by generate bandwidths enhancement from nano-ring resonator. Moreover, a PANDA ring resonator [6-8] is the optical filter powerfully by using an optical dark-bright soliton control arrangement within a semiconductor add-drop multiplexer, the promising applications have been observed [9]. In applications, one of the advantages is that the dark soliton peak signal always at low level, which is useful for secured signal communication in the transmission link. The other applications are associated to the high optical field configurations (as an optical tweezer or potential well) [10]. Spin manipulation has generated tremendous research progress in many fields, as light-shift effect in rubidium atom [11], magnetic clusters [12], semiconductors [13], GaAs/AlGaAs quantum well [14], carbon nanotubes [15], thin-film nanomagnets [16], magnetic tunnel junctions [17], and CdTe quantum dots [18]. All of them are based on pulsed magnetic resonance technique which is well developed, but to date the single spin detection still remains a challenging task. The use of photon states for spin manipulation has become a promising technique of investigation, in which photons are the ideal candidates to transmit quantum information with little de-coherency. Furthermore, spin states can be used to store and process quantum information due to their long coherence time. Therefore, the investigations of spin manipulation [19, 20], spin detection, remote spin entanglement mediated by photons, quantum state transfer between photons and spins are of great importance. However, there is still need to explore the aspect of spin coupling and orbital motion of electrons in carbon nanotubes, magnetic manipulations, sidewall oxide effects on 
spin torque, magnetic field induced reversal characteristics of thin film nanomagnets, magnetic vortex oscillator driven by direct spin polarized current and measurement of the spin transfer torque vector in magnetic tunnel junctions $[21,22]$.

In this paper, the use of a modified add-drop optical filter structure is analyzed within PANDA ring resonator system to form the orthogonal sets of solitons, which can be decomposed into left and right circularly rotated (polarized) waves. PANDA is a Chinese bear which is used to call a modified add-drop optical filter by the authors. This can be done relative to any orthogonal set of axes, where typical plane wave of ordinary light consists of components with all polarizations mixed together. The use of a new orthogonal set of light pulses called a dark-bright soliton pair [23] is proposed to form the two components of circularly rotated waves. In principle, the orthogonal solitons can be used to form the two components of polarized light, which are called the optoelectronic spin states. Finally, the different soliton spins are described by using several pairs of the orthogonal solitons, we proposed these properties using for optical wireless communication by applied the spin down for downlink and spin up for uplink.

\section{Theoretical Background}

A dark-bright soliton conversion system using a ring resonator optical channel dropping filter is composed of two sets of coupled waveguides, as shown in Fig. 1. The relative phase of the two output light signals after coupling into the optical coupler is $\pi / 2$. Thus, the signals coupled into the drop and through ports have acquired a phase of $\pi$ with respect to the input port signal. The resonant wavelength at through port on resonance can be extinguished completely by engineering the appropriate coupling coefficients and as a result all the power would be coupled into the drop port. The control fields at the input and add ports are formed by the dark and bright optical solitons and described by Equations (1) and (2), respectively $[5,7]$.

$$
\begin{aligned}
& E_{\text {in }}(t)=A_{0} \tanh \left[\frac{T}{T_{0}}\right] \exp \left[\left(\frac{z}{2 L_{D}}\right)-i \omega_{0} t\right] \\
& E_{\text {in }}(t)=A_{0} \operatorname{sech}\left[\frac{T}{T_{0}}\right] \exp \left[\left(\frac{z}{2 L_{D}}\right)-i \omega_{0} t\right]
\end{aligned}
$$

Here $A_{0}$ and $\mathrm{z}$ are the optical field amplitude and propagation distance, respectively. $T=t-\beta_{1} z$, where $\beta_{1}$ and $\beta_{2}$ are the coefficients of the linear and second-order terms of Taylor expansion of the propagation constant. $\mathrm{LD}=T_{0}^{2} /\left|\beta_{2}\right|$ is the dispersion length of the soliton pulse. T0 is a soliton pulse propagation time at initial input (or soliton pulse width), where $t$ is the soliton phase shift time, and $\omega_{0}$ is the frequency shift of the soliton. The optical fields of the system can be expressed as follow

$$
\begin{aligned}
& E_{1}=-j \kappa_{1} E_{i}+\tau_{1} E_{4}, \\
& E_{2}=\exp (j \omega T / 2) \exp (-\alpha L / 4) E_{1}, \\
& E_{3}=\tau_{2} E_{2}-j \kappa_{2} E_{a},
\end{aligned}
$$

$$
\begin{aligned}
& E_{4}=\exp (j \omega T / 2) \exp (-\alpha L / 4) E_{3}, \\
& E_{t}=\tau_{1} E_{i}-j \kappa_{1} E_{4}, \\
& E_{d}=\tau_{2} E_{a}-j \kappa_{2} E_{2},
\end{aligned}
$$

Here $E_{i}$ is the input field, $E_{a}$ is the add(control) field, $E_{t}$ is the through field, $E_{d}$ is the drop field, $E_{1} \ldots E_{4}$ are the fields in the ring at points $1 \ldots 4, \kappa_{1}$ is the field coupling coefficient between the input bus and ring, $\kappa_{2}$ is the field coupling coefficient between the ring and output bus, $L$ is the circumference of the ring, $T$ is the time taken for one round trip(roundtrip time), and $\alpha$ is the power loss in the ring per unit length. We assume that this is the lossless coupling, i.e., $\tau_{1,2}=\sqrt{1-\kappa_{1,2}^{2}}$. $T=L n_{\text {eff }} / c$.

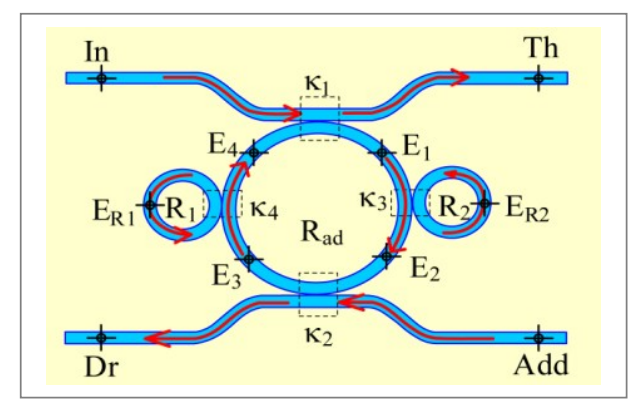

Fig. 1. A schematic diagram of an InGaAsP/InP PANDA ring resonator.

Figure 1, where $R_{i}$ : ring radii, $E_{i}$ : electric fields, $\kappa_{i}$ : coupling coefficients, In: input field, Th: through port, $D r$ : drop port, and Add: add port. The output intensities at the drop and through ports are given by equations 9 and 10, Where $A_{1 / 2}=\exp (-\alpha L / 4) \quad$ (the half-round-trip amplitude), $A=A_{1 / 2}^{2} \quad, \quad \Phi_{1 / 2}=\exp (j \omega T / 2) \quad$ (the half-round-trip phase contribution), and $\Phi=\Phi_{1 / 2}^{2}$.

$$
\begin{aligned}
\left|E_{d}\right|^{2} & =\left|\frac{-\kappa_{1} \kappa_{2} A_{1 / 2} \Phi_{1 / 2}}{1-\tau_{1} \tau_{2} A \Phi} E_{i}+\frac{\tau_{2}-\tau_{1} A \Phi}{1-\tau_{1} \tau_{2} A \Phi} E_{a}\right|^{2} . \\
\left|E_{t}\right|^{2} & =\left|\frac{\tau_{2}-\tau_{1} A \Phi}{1-\tau_{1} \tau_{2} A \Phi} E_{i}+\frac{-\kappa_{1} \kappa_{2} A_{1 / 2} \Phi}{1-\tau_{1} \tau_{2} A \Phi} E_{a}\right|^{2} .
\end{aligned}
$$

\section{Optical Spin Generation for OWLAN Simultaneous up-down link}

The optical wireless LAN operations, clients mobile communicate asynchronously by using some optical orthogonal codes such as optical CDMA are used as the signature sequence to separate the active client's information. Figure 7, simple Line of Sight (LoS) model for the optical channel, optical transmitters and receivers for example use Intensity Modulation/Direct Detection (IM/DD) technique with On-Off Keying (OOK) signaling for data communication. The optical wireless access point (OWAP), sent/received data from client node with single pair of optical transmitter and reviver or optical code (OC) for the downlink and uplink 
respectively. The downlink signal are the data stream of code together with driver and optical transmitter, then transmit to all client mobile nodes. On the other hand, the uplink channel all active clients mobile send their data bits asynchronously on the common channel and therefore uplink signal to all clients.

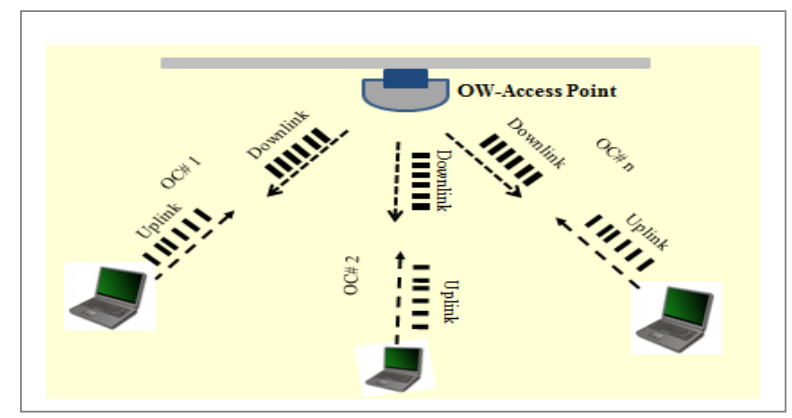

Fig. 2. A Optical wireless LAN communication uplink and downlink.

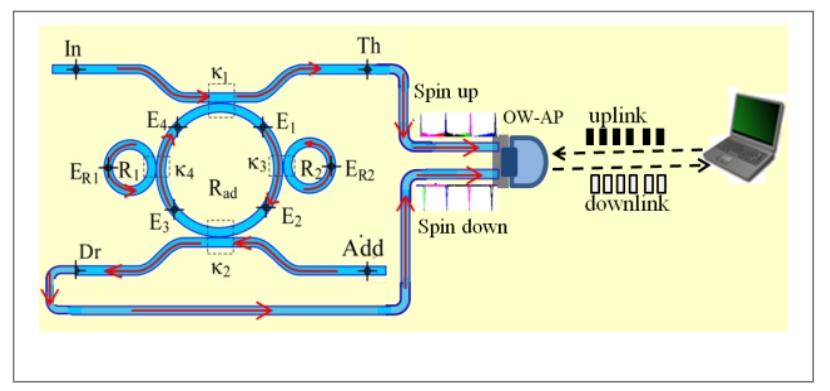

Fig. 3. A PANDA ring resonator for generate optical signal uplink down link.

The proposed of this article show in figure 3, applied the PANDA ring for generate the spin communication channels. The PADA ring embeds a part of optical wireless access point and generates spin-up and spin-down channel to support the simultaneous up-down links. From a picture, the output signal leak simultaneous from through port for spin up and drop port for spin down, then modulate signal by optical wireless access point for transmit to mobile client. The optical wireless access point modulates the spin down signal for downlink to transmit information to local optical station. On the opposite way received information from local optical wireless station by spin up signal for uplink. The method for uplink and downlink signal generation explore in bellow section.

\section{Simulation Results}

In operation, the orthogonal soliton sets can be generated by the proposed system as shown in Fig. 1. The optical field is fed into the PANDA system, where $R_{1}=R_{2}=2.5 \mu \mathrm{m}, R_{a d}=$ $30 \mu \mathrm{m}, A_{\text {eff }}=0.25 \mu \mathrm{m}^{2}, n_{\text {eff }}=3.14, n_{2}=1.3 \times 10^{-13} \mathrm{~cm}^{2} / W$ (for InGaAsP/InP waveguide)[24], $\alpha=0.1 \mathrm{~dB} / \mathrm{mm}$, gap coefficients, $\kappa_{1}=\kappa_{2}=0.5, \kappa_{3}=\kappa_{4}=0.3, \gamma=0.01, \lambda_{0}=1350,1450,1500$, 1550 , and $1600 \mathrm{~nm}$. It has been observed that when the dark soliton arrays are fed into a PANDA ring, the bright soliton conversion pulses are seen at $E_{1}$ and $E_{4}$ position (as shown figure 4(a), and (d)), and the dark soliton conversion pulses are seen at $E_{2}$ and $E_{3}$ position (as shown figure $4(\mathrm{~b})$ and (c)).
Figure 4, the different wavelengths are represented by different colors, where the horizontal axis represents wavelength. The wavelength-frequency domain conversion is formed by $\mathrm{c}=\mathrm{f} / \lambda$ to usual time domain representation in which a bright (dark) soliton pulse repeats itself in the propagation. The dynamic soliton circulated power within a PANDA ring resonator is shown in figure (5) and (6), with peaks (1), (2), (3) and (4) detected at positions E1, E2, E3 and E4 respectively. In the case of a large number of photons, the angular momentum of the solitons generated by a dark soliton pump based-on (port In) a PANDA ring resonator at center wavelength $1450 \mathrm{~nm}$ is essentially zero. When a soliton is absorbed by an object, an angular momentum of either $+\hbar$ or $\hbar$ is imparted to the object, in which two possible spin states known as optoelectronic (soliton) spins are exhibited, where this is due to fact that the half number of photons exhibit righthand and the rest half exhibit left-hand circularly motion, in which spin effect of each other due to the superposition of left and right circularly polarized solitons can be cancelled, which means that the spin conservation is maintained. The spatial solitons are generated within a PANDA ring resonator as shown in figure 6 . The peak (1) illustrates a R-hand spin with power $0.2 \times 10-4 \mathrm{~dB}, 0.3226 \mathrm{~ns}$, peak (2) R-hand spin with power $5 \times 10-3 \mathrm{~dB}, 0.5081 \mathrm{~ns}$, peak (3) L-hand spin with power $8.5 \times 10-3 \mathrm{~dB}, 0.7526 \mathrm{~ns}$ at the drop port and peak (4) L-hand spin with power $9.5 \times 10-3 \mathrm{~dB}, 0.9459 \mathrm{~ns}$ at the through port.

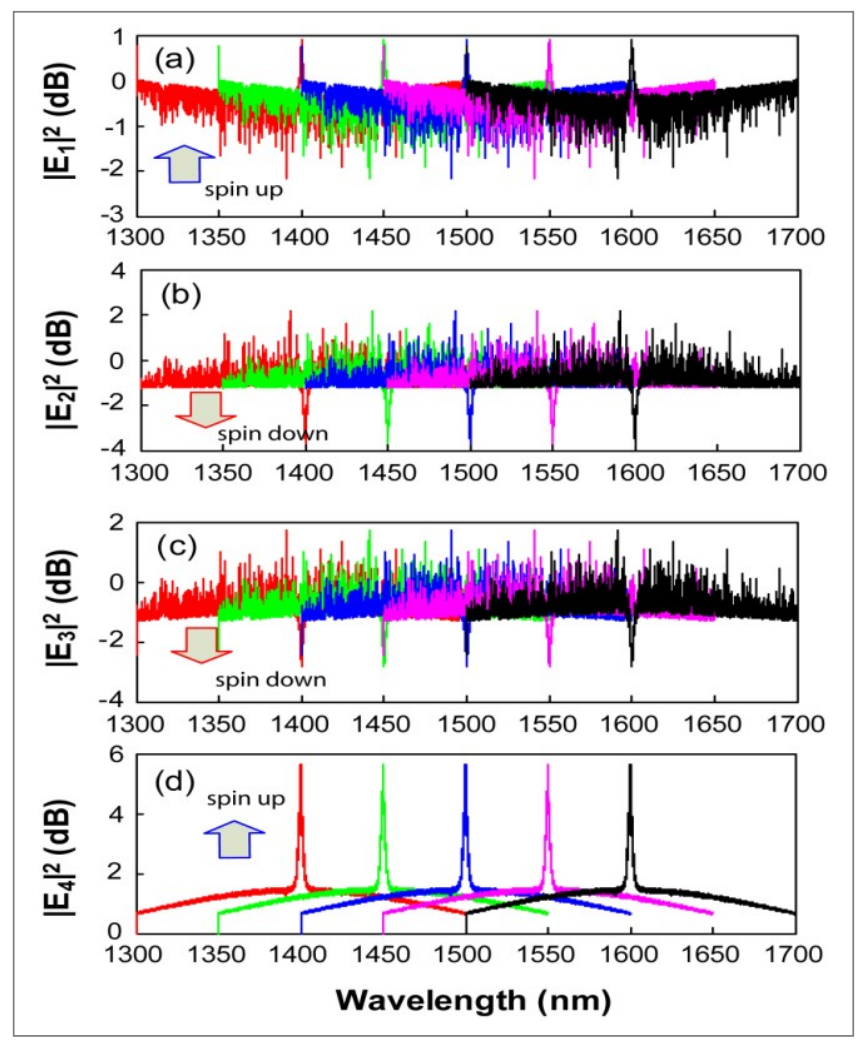

Fig. 4. Many soliton spins within a PANDA ring are generated by using a dark-soliton pump input port; $R_{1}=R_{2}=2.5 \mu \mathrm{m}, R_{a d}=30 \mu \mathrm{m}$; center wavelength $1350,1450,1500,1550$, and $1600 \mathrm{~nm}$. 
The spatial solitons are generated within a PANDA ring resonator as shown in figure 6 . The peak (1) illustrates a Rhand spin with power $0.2 \times 10-4 \mathrm{~dB}, 0.3226 \mathrm{~ns}$, peak (2) Rhand spin with power $5 \times 10-3 \mathrm{~dB}, 0.5081 \mathrm{~ns}$, peak (3) L-hand spin with power $8.5 \times 10-3 \mathrm{~dB}, 0.7526 \mathrm{~ns}$ at the drop port and peak (4) L-hand spin with power $9.5 \times 10-3 \mathrm{~dB}, 0.9459 \mathrm{~ns}$ at the through port. In figure 6 , the spatial mode structures are shown by peaks $1,2,3$, and 4 , where they are the superposition of the soliton pairs at the positions E1, E2, E3 and E4. The spatial mode peak 1 is the spin state of a single soliton pair after passing the first coupler, $\kappa_{1}$. The spatial mode peaks 2, 3 and 4 are the spin states after passing the microring, $R 2$, second coupler, $\kappa_{2}$ and microring, $R 1$, respectively.

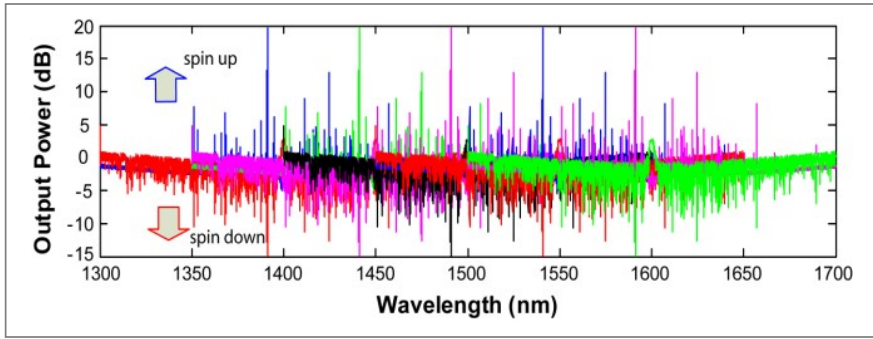

Fig. 5. Many spin solitons are detected at through (spin up) and drop (spin down) ports of a PANDA ring resonator.

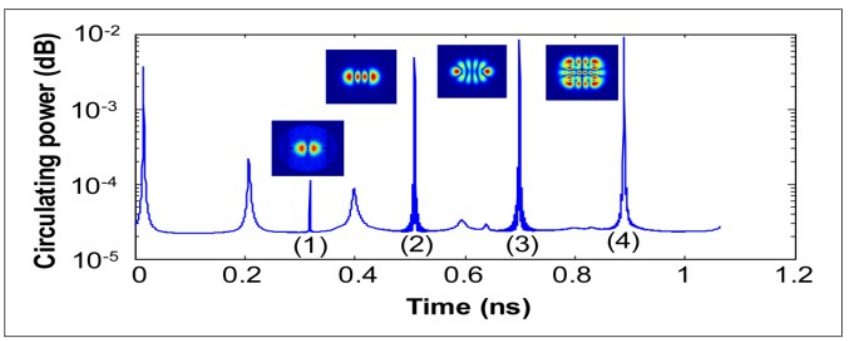

Fig. 6. Many soliton spins are circulated within a PANDA ring resonator, where (1) $R$-hand spin detected at $\mathrm{E}_{1}$, (2) $R$-hand spin detected at $\mathrm{E}_{2}$, (3) $L$-hand spin detected at $E_{3}$, and (4) $L$-hand detected at $E_{4}$, positions, respectively.

In applications, the output signals (orthogonal solitons or photons) corresponding to the left-hand and right-hand photons can be generated and detected randomly at the same wavelength via the Th and Dr ports as shown in figure 5. The angular momentum of either $+\hbar$ or $-\hbar$ is imparted to the object when a photon is absorbed by an object. Thus, the array of soliton spins can be generated and controlled by the proposed system, which is available for high density spin states use. The spiral phase structure in a PANDA ring system causes the soliton pulse rotation for the right and left microrings faster than center ring $R$-hand and $L$-hand.

\section{Conclusion}

In this paper, design the new simultaneous optical wireless up-down link by using optical spin. The spin is orthogonal sets and manipulated by using the orthogonal soliton pair within a PANDA ring resonator known as a dark-bright soliton conversion pulses. In this manipulation, the electromagnetic radiations were generated by using the orthogonal soliton pulses. Soliton is a light pulse which will be detected at the output ports (through or drop ports), where finally the energy conversion between soliton (light) and photon (particle) can be observed by using the optical detector via the output ports. Hence, the spin axis of photon is always parallel to its direction of motion. The orthogonal sets are available, in which the spin conservation of many particles is maintained for OWLAN uplink and downlink simultaneously.

\section{References}

[1] J. B. Carruthers, "Wireless infrared communications", online. J Wiley Ency. of Telecom, APR 2003.

[2] S. Khazraei, M. R. Pakravan, "Analysis of generalized optical orthogonal codes in optical wireless local area networks", IEEE J. ON sele. area in comm., vol. 27, no.12, Dec. 2008, pp.2145-2155.

[3] B. M. Ghaffari,M. D. Matinfar, and J. A. Salehi, "Wireless optical CDMA LAN: digital design concepts", IEEE Trans. on Comm., vol. 56, Dec. 2008,pp.2145-2155.

[4] S. Mitatha, M. Bunruangses, K. Sunat, and P. P. Yupapin, "Novel vanet using a simultaneous wireless up-down link", Micro. and Opti. Tech. Lett., vol. 52, no.8, May. 2010, pp.1764-1768.

[5] S. Mitatha, P. P. Yupapin, "Fast and slow light generated simultaneously for up-down-link frequency converter applications", J. Optik, vol. 121, no.14, Aug. 2010, pp.1313-1316.

[6] T. Phatharaworamet, C. Teeka, R. Jomtarak, S. Mitatha, and P.P. Yupapin, "Random binary code generation using dark-bright soliton conversion control within a PANDA ring resonator", J. Lightw. Technol. Vol. 28, No.2804, Oct. 2010, pp.2804-2809.

[7] N. Suwanpayak, M.A. Jalil, C. Teeka, J. Ali, and P.P. Yupapin, "Optical vortices generated by a PANDA ring resonator for drug trapping and delivery applications", Biomed. Opt. Express,vol. 2, no.1,Jan. 2011,pp.159-168.

[8] P. Youplao, T. Phattaraworamet, S. Mitatha, C. Teeka, and P.P. Yupapin, "Novel optical trapping tool generation and storage controlled by light", J. Nonlin. Opt. Phys. Mater., Vol. 19, No. 02, June 2010, pp. 371-378.

[9] K. Sarapat, N. Sangwara, K. Srinuanjan, P.P. Yupapin, and N. Pornsuwancharoen, "Novel dark-bright optical solitons conversion system and power amplification", Opt. Eng., Vol.48, No. 4. Apr.2009.

[10] B. Piyatamrong, K. Kulsirirat, W. Techithdeera, S. Mitatha, and P.P. Yupapin, "Dynamic potential well generation and control using double resonators incorporating in an add/drop filter", Mod. Phys. Lett., Vol. 24, No. 32, Dec. 2011, pp. 3071-3080.

[11] T. Moriyasu, D. Nomoto, Y. Koyama, Y. Fukuda, and T. Kohmoto, "Spin manipulation using the light-shift effect in rubidium atoms", Phys. Rev. Lett. 103, Nov 2009.

[12] W. Hübner, S. Kersten, and G. Lefkidis, "Optical spin manipulation for minimal magnetic logic operations in metallic three-center magnetic clusters", Phys. Rev., Vol.79, No.18, May 2009.

[13] R.C. Myers, M.H. Mikkelsen, J.-M. Tang, A.C. Gossard, M.E. Flatte, and D.D. Awschalom, "Zero-field optical manipulation of magnetic ions in semiconductors", Nature Matter.,Vol.7, Feb. 2008, pp. 203-208.

[14] F. Kuemmeth, S. Ilani, D.C. Ralph, and P.L. McEuen, "Coupling of spin and orbital motion of electrons in carbon nanotubes", Lett. Of Nature, Vol.452, Feb.2008,pp.448-452.

[15] T.Kom, "Time-resolved studies of electron and hole spin dynamics in modulation-doped GaAs/AlGaAs quantum wells", J. of ELSEVIER, Vol. 494, No. 5, Sep. 2010,pp. 415-445.

[16] O. Ozatay, P.G. Gowtham, K.W. Tan, J.C. Read, K.A. Mkhoyan, M.G. Thomas, G.D. Fuchs, P.M. Braganca, E.M. Ryan, K.V. Thadan, J. Silcox, D.C. Ralph, and R.A. Buhrman, "Sidewall oxide effects on spintorque- and magnetic-field-induced reversal characteristics of thin-film nanomagnets", Nature Mater., Vol.7, Junn 2008, pp. 567-573. 
[17] D. F. Gordon, B. Hafizi, and A. Ting, "Nonlinear conversion of photon spin to photon orbital angular momentum", Opt. Lett., Vol. 34, No.34, Nov. 2009,pp. 3280-3282.

[18] M. Goryca, T. Kazimierczuk, M. Nawrocki, A. Golnik, J.A. Gaj, P. Wojnar, G. Karczewski, and P. Kossacki, "Optical manipulation of a single Mn spin in a CdTe quantum dot", J. of ELSEVIER, Vol. 42, No. 10, Sep. 2010,pp.2690-2693.

[19] R. I. Shekhter, A. M. Kadigrobov, M. Jonson, E. I. Smotrova, A. I. Nosich, and V. Korenivski, "Subwavelength terahertz spin-flip laser based on a magnetic point-contact array", Opt. Lett., Vol. 36, No.12, Jun. 2011,pp. 2381-2383.

[20] V.S. Pribiag, I.N. Krivorotov, G.D. Fuchs, P.M. Braganca, O. Ozatay, J.C. Sankey, D.C. Ralph, and R.A. Buhrman,"Magnetic vortex oscillator driven by d.c. spin-polarized current", Nature Phys., Vol. 3, May 2007,pp. 498-503.

[21] O. M. J. Erve, C. Awo-Affouda, A.T. Hanbicki, C.H. Li, P.E. Thompson, and B.T. Jonker, "Information processing with pure spin currents in silicon: spin injection, extraction, manipulation, and detection", IEEE Transac. Electron Dev., Vol.56, No. 10, Oct. 2009,pp.2343-2347.

[22] E. Brasselet, Y. Izdebskaya, V. Shvedov, A. S. Desyatnikov, W. Krolikowski, and Y. S. Kivshar, "Dynamics of optical spin-orbit coupling in uniaxial crystals", Opt. Lett., Vol. 34, No.7, Apr. 2009, pp. 1021-1023.

[23] Y. Zhang, Z. Wang, Z. Nie, C. Li, H. Chen, K. Lu, and M. Xiao, "FourWave Mixing Dipole Soliton in Laser-Induced Atomic Gratings", Phys. Rev. Lett., Vol. 106, No. 9, Sep. 2011.

[24] J. Zhu, S.K. Ozdemir, Y.F. Xiao, L. Li, L. He, D.R. Chen, L. Yang, "On-chip single nanoparticle detection and sizing by mode splitting in an ultrahigh- $Q$ microresonator", Nat. Photon., Vol. 4, Dec. 2010, pp. 46-49. 